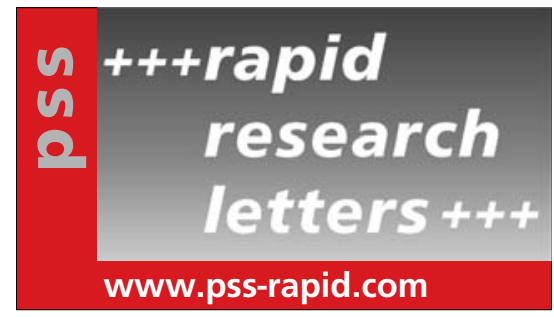

\title{
Femtosecond laser micromachined optical waveguides in $\mathrm{LiTaO}_{3}$ crystal
}

\author{
Miaomiao Xu**, ${ }^{*, 2}$, Ruiyun $\mathrm{He}^{1}$, Shuqian Sun ${ }^{1,2}$, Javier R. Vázquez de Aldana ${ }^{3}$, and Feng Chen ${ }^{* 1}$ \\ ${ }^{1}$ School of Physics, State Key Laboratory of Crystal Materials and Key Laboratory of Particle Physics and Particle Irradiation \\ (Ministry of Education), Shandong University, Jinan 250100, P.R. China \\ ${ }^{2}$ Taishan College, Shandong University, Jinan 250100, P.R. China \\ ${ }^{3}$ Laser Microprocessing Group, Universidad de Salamanca, Salamanca 37008, Spain
}

Received 31 July 2013, revised 23 August 2013, accepted 26 August 2013

Published online 28 August 2013

Keywords optical waveguides, $\mathrm{LiTaO}_{3}$, crystals, laser micromachining

\footnotetext{
* Corresponding author: e-mail drfchen@sdu.edu.cn, Phone: +86-531-88363007, Fax: +86-531-88363350

** e-mail mmxu1992@gmail.com, Phone: +86-531-88363496
}

By using femtosecond laser micromachining, optical wave guides in both depressed cladding and dual-line configurations have been produced in $\mathrm{LiTaO}_{3}$ crystal. The guiding properties and the thermal stability have been investigated for both geometries, which exhibit different performance. Depressed cladding waveguides support guidance along both ex- traordinary and ordinary index polarizations, while dual-line waveguides support only extraordinary index polarization. Thermal annealing has been proved to be an effective method to reduce the propagation losses. For the cladding waveguide, the lowest propagation loss was as low as $0.38 \mathrm{~dB} / \mathrm{cm}$ after the annealing treatment at $400{ }^{\circ} \mathrm{C}$.
1 Introduction In integrated photonics, the optical waveguides are one of the basic components which can confine the light propagation and supply high optical intensity. So far, different techniques have been developed to produce optical waveguides, such as thermal ion in diffusion [1], ion implantation/irradiation [2], ion exchange [3] and femtosecond (fs) laser micromachining [4]. As a unique technique which can provide high spatial resolution in three-dimensional (3D) micro-structuring, fs-laser micromachining has been used to fabricate optical waveguides in various transparent materials, including glasses, single crystals, ceramics, and polymers [5-17]. By tightly focusing the fs-laser beams into the bulk of the transparent material, the refractive index modification of a small volume can be induced. The performances of these waveguides depend not only on the properties of the material, but also on the parameters of the micromachining, such as the pulse duration and energy, the repetition rate, polarization, focal depth, or the scan speed [11-14]. There are several types of fs-laser fabricated optical waveguides depending on the inscription strategy. In Type I waveguides, the fs-laser irradiated area exhibits a refractive index increase, whereas in Type II waveguides a severe damage track is produced in the material that leads to a refractive index de- crease at the irradiated area [15] with a slight increase in the vicinity of the track. For the fabrication of these waveguides, two parallel laser scans are done (dual line) and the confining region appears between the two damage tracks due to the stress produced in the material. In addition, a depressed cladding waveguide can be fabricated by the fslaser inscription as well, whose guiding core is normally surrounded by a number of low-index tracks [15-17]. In a $3 \mathrm{D}$ view, the cladding waveguides look like photonic tubes typically with circular cross-sectional shapes, and arbitrary diameters (typically from $30 \mu \mathrm{m}$ to $200 \mu \mathrm{m}$ ). Since the circular-shape cross section with desired diameter can be designed to fit well to that of an optical fibre, these cladding structures seem to be promising to realize the integration of fibre-waveguide photonic systems.

Lithium tantalate $\left(\mathrm{LiTaO}_{3}\right)$ is an excellent crystal for various nonlinear and electrooptic applications [18]. It has better enduring ability for photorefraction compared with the more commonly used $\mathrm{LiNbO}_{3}$, with an optical refraction threshold three times higher than the latter one [19, 20]. This makes it having special applications in several fields, including nuclear physics, space physics and nuclear medicine. In early works, the $\mathrm{LiTaO}_{3}$ waveguides were fabricated by proton exchange [21], and guiding character- 
istic as well as the effect of annealing were investigated. Although fs-laser micromachining of $\mathrm{LiTaO}_{3}$ has been realized [22], and the nonlinear optical properties have been investigated [23], the guiding properties of $\mathrm{LiTaO}_{3}$ waveguides have not been reported until now.

In this work, we report on the fabrication of depressed cladding waveguides and dual-line (Type II) waveguides in $\mathrm{LiTaO}_{3}$ crystal produced by fs-laser micromachining. The optical guiding properties in terms of the light polarization and the thermal stability are also investigated.

2 Experimental details The $z$-cut $\mathrm{LiTaO}_{3}$ crystal was cut into $15 \times 10 \times 1 \mathrm{~mm}^{3}$ pieces and optically polished. Four depressed cladding waveguides (WG1-WG4) and two Type II waveguides (WG5 and WG6) with dual-line structures were inscribed parallel to the $10 \mathrm{~mm}$ side boundary under the surface $\left(15 \times 10 \mathrm{~mm}^{2}\right)$. A Ti:sapphire laser system was used to generate $120 \mathrm{fs}$ pulses, linearly polarized at $800 \mathrm{~nm}$ and with a low repetition rate of $1 \mathrm{kHz}$. The sample was located on a 3D motorized stage and one $20 \times$ microscope objective (N.A. $=0.40$ ) was used to focus the laser at the depth of $\sim 150 \mu \mathrm{m}$ under the largest sample surface. The sample was scanned with different velocities $(500 \mu \mathrm{m} / \mathrm{s}, 200 \mu \mathrm{m} / \mathrm{s}, 500 \mu \mathrm{m} / \mathrm{s}, 500 \mu \mathrm{m} / \mathrm{s}, 20 \mu \mathrm{m} / \mathrm{s}$, and $50 \mu \mathrm{m} / \mathrm{s}$ for WG1-WG6, respectively). A calibrated neutral density filter, a half-wave plate and a linear polarizer were used to control the incident pulse energy that was set to $2.9 \mu \mathrm{J}$ for all the waveguides. The detailed fabrication procedure is explained elsewhere (see [15] for instance).

A polarized microscope (Axio Imager, Carl Zeiss) was used to photograph the cross sections of the fabricated waveguides. The study of the near-field modal profiles was performed by utilizing a typical end-face arrangement. We used a pair of microscope objective lenses $(25 \times)$ to couple a $\mathrm{He}-\mathrm{Ne}$ laser $(632.8 \mathrm{~nm})$ into and out of the waveguides. Afterwards, the images were collected by a CCD camera, and analysed by the RayCi software. To calculate the propagation losses of the $\mathrm{LiTaO}_{3}$ waveguides, we measured the input and output light power of the waveguides, and estimated the coupling loss and the Fresnel reflection in the interface of the waveguide end face and the air. Finally, to calculate the index change, a cuboid glass was located at a rotary indexing table to measure the maximum angle difference. Afterwards, the sample was annealed by an open oven. The guiding properties and propagation losses were again measured after each annealing. In this work, we annealed twice, at $260{ }^{\circ} \mathrm{C}$ and $400{ }^{\circ} \mathrm{C}$ in sequence, both for $30 \mathrm{~min}$. Here we labelled them as annealing treatments Step 1 and Step 2 separately. After Step 2, we used a $1064 \mathrm{~nm}$ laser to investigate the near-field modal distributions of the waveguides in the infrared regime.

\section{Results and discussion}

3.1 Waveguide configurations Figure 1 shows the images of the waveguides WG1-WG6. As one can see, the cladding waveguide cores were located in the regions surrounded by parallel fs-laser inscribed tracks with the adja-

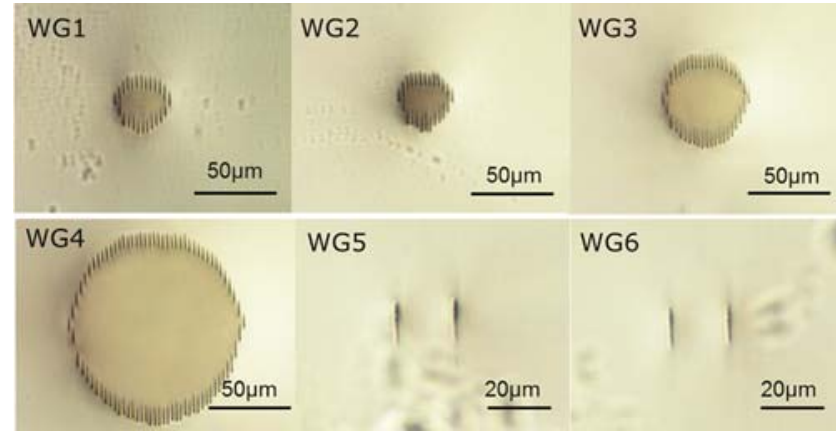

Figure 1 Optical microscope images of the cross sections of the depressed cladding waveguides (WG1-WG4) and the dual-line waveguides (WG5 and WG6).

cent separation of $\sim 3 \mu \mathrm{m}$. The diameters of the four waveguides were $\sim 30 \mu \mathrm{m}, \sim 30 \mu \mathrm{m}, \sim 50 \mu \mathrm{m}$ and $\sim 110 \mu \mathrm{m}$, respectively. The cores of the two dual-line waveguides were located in the regions between two parallel damage tracks with a separation of $20 \mu \mathrm{m}$. Pictures of the cross sections were taken after each annealing process. The structure of the waveguides had no obvious change after annealing, which showed that $\mathrm{LiTaO}_{3}$ waveguides had good thermal stability.

3.2 Modal profiles $\mathrm{The}_{\mathrm{LiTaO}}$ sample was $z$-cut, which suggested that the TM modal distributions correspond to polarization along the extraordinary index $\left(n_{\mathrm{e}}\right)$, while the TE modal distributions correspond to the ordinary index $\left(n_{0}\right)$ polarization. Figure 2 (top) shows the nearfield modal distributions for the $n_{\mathrm{e}}$ and $n_{\mathrm{o}}$ polarizations at $632.8 \mathrm{~nm}$. One can see that the cladding waveguides had similar modal profiles along the two polarizations. They presented multimode behaviour both in waveguides with small cross sections (WG1 and WG2) and in those with bigger ones (WG3 and WG4). After annealing at $400{ }^{\circ} \mathrm{C}$

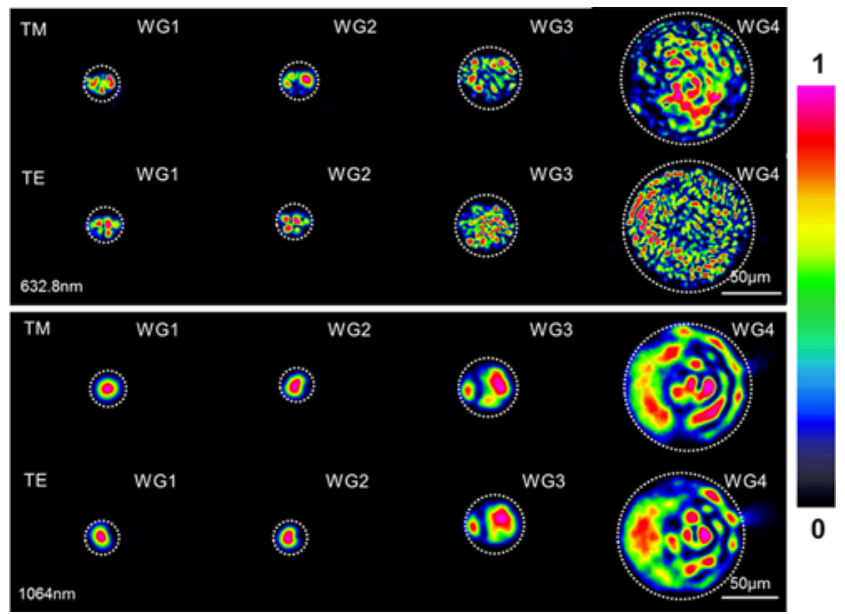

Figure 2 Measured near-field intensity distributions for the TM and TE modes of the cladding waveguides WG1-WG4 at $632.8 \mathrm{~nm}$ (top), and at $1064 \mathrm{~nm}$ after $400{ }^{\circ} \mathrm{C}, 30 \mathrm{~min}$ annealing (bottom). 

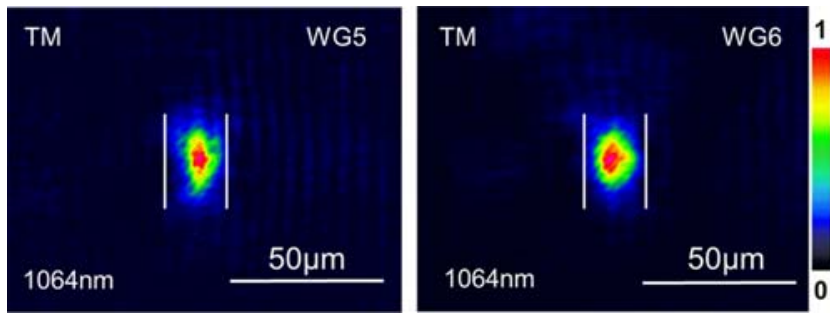

Figure 3 Measured near-field intensity distributions of the two dual-line waveguides WG5 and WG6 for TM polarization at $1064 \mathrm{~nm}$ after $400{ }^{\circ} \mathrm{C}, 30 \mathrm{~min}$ annealing process.

for $30 \mathrm{~min}$, near-field modal profiles along both polarizations were measured at $1064 \mathrm{~nm}$. WG1 and WG2 turned into single-modes, and the number of modes in WG3 and WG4 was obviously reduced (Fig. 2, bottom). Such phenomenon is quite accordant with the theory of guidedwave optics.

Before the annealing treatment, no guided light was observed for $n_{\mathrm{o}}$ or $n_{\mathrm{e}}$ polarizations of the dual-line waveguides WG5 and WG6. After Step 1, though some light was guided for the $n_{\mathrm{e}}$ polarization, its property for confining light was poor as some parasitic light appeared around the emergent light. After Step 2, single-mode profiles for $n_{\mathrm{e}}$ polarization were achieved, as shown in Fig. 3 , but we still could not figure out the modal profile for $n_{\mathrm{o}}$ polarization.

3.3 Refractive index profiles We used the numerical aperture method [17] to estimate the refractive index contrasts between the edge and the core of the waveguides. We calculated the maximum acceptance angle $\theta_{\mathrm{m}}$. Then the maximum index contrast was calculated by

$$
\Delta n \approx \frac{\sin ^{2} \theta_{\mathrm{m}}}{2 n}
$$

where $n$ is the original index of $\mathrm{LiTaO}_{3}$ crystal. We calculated the maximum refractive index contrast after each annealing. For the waveguides WG1-WG4, the maximum refractive-index changes at $632.8 \mathrm{~nm}$ for $n_{\mathrm{e}}(\mathrm{TM})$ modes before annealing were $4.5 \times 10^{-4}, 4.2 \times 10^{-4}, 8.5 \times 10^{-4}$, $12.8 \times 10^{-4}$, which were nearly equal to the index change for $n_{\mathrm{o}}$ modes: $4.6 \times 10^{-4}, 4.7 \times 10^{-4}, 8.4 \times 10^{-4}, 12.5 \times 10^{-4}$, respectively. We observed that these values had a notable increase after Step 1, but Step 2 made them to drop back nearly to the initial values. For the dual-line waveguides WG5 and WG6, the data of maximum refractive-index contrast before Step 2 were $4.2 \times 10^{-4}$ and $3.3 \times 10^{-4}$ for $n_{\mathrm{e}}$ polarization, yet these values dropped slightly after the annealing at $400{ }^{\circ} \mathrm{C}$.

By using the data of $\Delta n$, the $2 \mathrm{D}$ refractive index profiles could be reconstructed with the same technique introduced in previous works $[24,25]$. We simulated WG1 at $1064 \mathrm{~nm}$ provided that it showed a good single-mode profile, shown in Fig. 4(a). Afterwards, we simulated the light propagation in the waveguide by using the computer soft
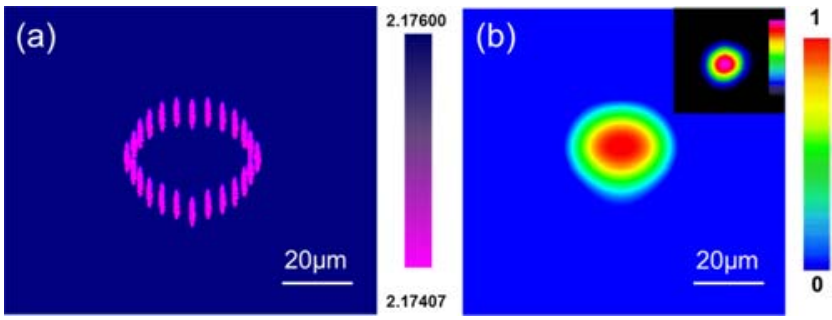

Figure 4 (a) Reconstructed 2D refractive index profile and (b) calculated modal profile of the cladding waveguide WG1 for TM polarization at $1064 \mathrm{~nm}$. The insert figure indicates the experimental result.

ware Rsoft based on the finite difference beam propagation method (FD-BPM) [24, 26]. Figure 4(b) shows the calculated modal profile of WG1. One can see that the calculated profile was in agreement with the experimental result, which proved that the reconstructed refractive index profile of the cladding waveguide was reasonable.

3.4 Propagation losses The propagation losses of waveguides are listed in Table 1. For the circular cladding waveguides WG1-WG4, the propagation losses for $n_{\mathrm{e}}$ polarization before annealing treatment were much higher than along the $n_{\mathrm{o}}$ polarization. These values had no significant change after the annealing treatment Step 1, showing thermal stability of cladding waveguides at $260{ }^{\circ} \mathrm{C}$. However, after annealing Step 2, the propagation losses of WG1-WG4 declined abruptly, as shown in Table 1. This effect may be because the annealing removes somehow the lattice defects induced during the inscription process, leading to an even larger decrease of the propagation losses.

For the dual-line waveguides WG5 and WG6, the propagation losses $\left(n_{\mathrm{e}}\right)$ of WG5 and WG6 were $10.82 \mathrm{~dB} / \mathrm{cm}$ and $16.58 \mathrm{~dB} / \mathrm{cm}$ after Step 1. Step 2 made the propagation losses much lower, as the values of WG5 and WG6 dropping down to $10.03 \mathrm{~dB} / \mathrm{cm}$ and $10.08 \mathrm{~dB} / \mathrm{cm}$.

Table 1 Propagation losses $\alpha$ of $\mathrm{LiTaO}_{3}$ waveguides WG1-WG6 for $n_{\mathrm{e}}$ and $n_{\mathrm{o}}$ polarizations at $632.8 \mathrm{~nm}$ before and after annealing treatments.

\begin{tabular}{lllrc}
\hline \multicolumn{6}{l}{ propagation losses $\alpha(\mathrm{dB} / \mathrm{cm})$} \\
\hline \multirow{2}{*}{ WG1 } & $n_{\mathrm{e}} / n_{\mathrm{o}}$ & before annealing & Step 1 & Step 2 \\
& $n_{\mathrm{e}}$ & 12.14 & 12.10 & 2.08 \\
WG2 & $n_{\mathrm{o}}$ & 3.87 & 3.91 & 1.73 \\
& $n_{\mathrm{e}}$ & 15.09 & 15.16 & 2.77 \\
& $n_{\mathrm{o}}$ & 4.95 & 5.00 & 2.41 \\
WG3 & $n_{\mathrm{e}}$ & 7.89 & 7.87 & 0.99 \\
& $n_{\mathrm{o}}$ & 1.58 & 1.54 & 0.62 \\
WG4 & $n_{\mathrm{e}}$ & 2.62 & 2.65 & 0.38 \\
& $n_{\mathrm{o}}$ & 0.70 & 0.72 & 0.49 \\
WG5 & $n_{\mathrm{e}}$ & - & 10.82 & 10.03 \\
WG6 & $n_{\mathrm{e}}$ & - & 16.58 & 10.08 \\
\hline
\end{tabular}


From Table 1, we could clearly see that the fabrication parameters had some influences on the properties of waveguides. The three cladding waveguides WG1, WG3 and WG4, which were fabricated at the same scanning velocity but with different diameters $\left(D_{1}<D_{3}<D_{4}\right)$, exhibit different propagation losses $\alpha\left(\alpha_{1}>\alpha_{3}>\alpha_{4}\right)$. This means that the propagation losses decrease by enlarging the radius of the circular cross section.

WG1 and WG2 had the same diameter but different scanning velocities were used during their fabrication $(500 \mu \mathrm{m} / \mathrm{s}$ and $200 \mu \mathrm{m} / \mathrm{s}$, respectively). Our measurements showed that WG1 had lower losses than WG2. In this work, the repetition rate of the laser was $1 \mathrm{kHz}$, which meant that the time between pulses was long enough so that the thermal diffusion had carried the laser-induced heat away from the focus before the next pulse arrives and the ensuing pulses act independently of one another. Thus, the larger losses measured at WG2 cannot be attributed to a stronger damage produced at the waveguide, but to a higher density of defects due to a larger spatial overlap between consecutive pulses. However, the dual-line waveguides WG5 and WG6, with the same separation between the damage tracks $(20 \mu \mathrm{m})$, showed the opposite correlation: as the scan velocity gets faster $\left(V_{5}=20 \mu \mathrm{m} / \mathrm{s}, V_{6}=50 \mu \mathrm{m} / \mathrm{s}\right)$, the propagation loss becomes higher $\left(\alpha_{5}<\alpha_{6}\right)$. In this case, we think that smaller velocities increase the magnitude of the stress field created in the material (larger spatial overlap), leading to a larger magnitude of the refractive index change.

In the studies presented in this work, the propagation losses and the refractive-index contrast decreased for the Step 2 annealing process. In general, one would expect that a waveguide with larger refractive-index contrast should confine the light better and have lower propagation losses, but in our case, the presence of defects (that induce scattering or absorption) changes this behaviour.

4 Conclusion We report on the fabrication of cladding and dual-line waveguides in $\mathrm{LiTaO}_{3}$ crystal by fs-laser micromachining. Cladding waveguides present good guiding properties both for the $n_{\mathrm{o}}$ and $n_{\mathrm{e}}$ polarizations at $632.8 \mathrm{~nm}$ and $1064 \mathrm{~nm}$. Dual-line waveguides exhibit worst performance: they confine the $n_{\mathrm{e}}$ polarized light only after proper thermal annealing $\left(400^{\circ} \mathrm{C}\right)$. This thermal treatment has positive effects for both cladding and dual-line waveguides. In particular, the propagation losses of cladding waveguides decrease to a value as low as $\sim 0.38 \mathrm{~dB} / \mathrm{cm}$ at $632.8 \mathrm{~nm}$. Our research indicates the promising applications of fs-laser micromachining $\mathrm{LiTaO}_{3}$ waveguides in integrated optics.

Acknowledgements This work was supported by the National Natural Science Foundation of China (No. 11274203), the Spanish Ministerio de Ciencia e Innovación (Projects CSD2007-00013 and FIS2009-09522), and Junta de Castilla y León (Project SA086A12-2).

\section{References}

[1] D. Jaque, E. Cantelar, and G. Lifante, Appl. Phys. B 88, 201-204 (2007).

[2] F. Chen, Laser Photon. Rev. 6, 622-640 (2012).

[3] A. Tervonen, B. R. West, and S. Honkanen, Opt. Eng. 50, 071107 (2011).

[4] R. R. Gattass and E. Mazur, Nature Photon. 2, 219-225 (2008).

[5] L. B. Fletcher, J. J. Witcher, N. Troy, S. T. Reis, R. K. Brow, and D. M. Krol, Opt. Express 19, 7929-7936 (2011).

[6] Y. Tan, Y. C. Jia, F. Chen, J. R. Vázquez de Aldana, and D. Jaque, J. Opt. Soc. Am. B 28, 1607-1610 (2011).

[7] A. Rodenas and A. K. Kar, Opt. Express 19, 17820-17833 (2011).

[8] T. Calmano, J. Siebenmorgen, O. Hellmig, K. Petermann, and G. Huber, Appl. Phys. B 100, 131-135 (2010).

[9] T. Calmano, A. G. Paschke, J. Siebenmorgen, S. T. Fredrich-Thornton, H. Yagi, K. Petermann, and G. Huber, Appl. Phys. B 103, 1-4 (2011).

[10] L. Kallepalli, V. Soma, and N. Desai, Opt. Eng. 51, 073402 (2012).

[11] J. Siebenmorgen, K. Petermann, G. Huber, K. Rademaker, S. Nolte, and A. Tünnermann, Appl. Phys. B 97, 251-255 (2009).

[12] S. M. Eaton, H. Zhang, M. L. Ng, J. Li, W. J. Chen, S. Ho, and P. R. Herman, Opt. Express 16, 9443-9458 (2008).

[13] M. Will, S. Nolte, B. N. Chichkov, and A. Tünnermann, Appl. Opt. 41, 4360-4364 (2002).

[14] M. Ams, G. D. Marshall, and M. J. Withford, Opt. Express 14, 13158-13163 (2006).

[15] F. Chen and J. R. Vázquez de Aldana, Laser Photon. Rev., DOI 10.1002/lpor.201300025 (2013).

[16] D. G. Lancaster, S. Gross, H. Ebendorff-Heidepriem, K. Kuan, T. M. Monro, M. Ams, A. Fuerbach, and M. J. Withford, Opt. Lett. 36, 1587-1589 (2011).

[17] A. G. Okhrimchuk, A. V. Shestakov, I. Khrushchev, and J. Mitchell, Opt. Lett. 30, 2248-2250 (2005).

[18] A. Ashkin, G. D. Boyd, J. M. Dziedzic, R. G. Smith, A. A. Ballman, J. J. Levinstein, and K. Nassau, Appl. Phys. Lett. 9, 72-74 (1966).

[19] R. Hsu, E. N. Maslen, D. Du Boulay, and N. Ishizawa, Acta Crystallogr. B 53, 420-428 (1997).

[20] Z. Y. Zhang, Y. Y. Zhu, H. F. Wang, L. C. Wang, S. N. Zhu, and N. B. Ming, Physica B 398, 151-158 (2007).

[21] K. Tada, T. Murai, T. Nakabayashi, T. Iwashima, and T. Ishikawa, Jpn. J. Appl. Phys. 26, 503 (1987).

[22] D. W. Ward, E. R. Statz, and K. A. Nelson, Appl. Phys. A 86, 49-54 (2007).

[23] B. McMillen, K. P. Chen, H. An, S. Fleming, V. Hartwell, and D. Snoke, Appl. Phys. Lett. 93, 111106 (2008).

[24] RSoft Design Group, Computer Software BeamPROP, http://www.rsoftdesign.com.

[25] Q. An, Y. Ren, Y. Jia, J. R. Vázquez de Aldana, and F. Chen, Opt. Mater. Express 3, 466-471 (2013).

[26] D. Yevick and W. Bardyszewiski, Opt. Lett. 17, 329-330 (1992). 